\title{
Healthbot.net: Patient Education with a Natural Speaking Robot before Catheter Ablation: Results from 47 Patients
}

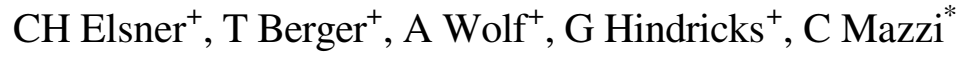 \\ ${ }^{+}$Heart Center Leipzig, Leipzig, Germany and *INSEAD Business School Fontainebleau, France
}

\begin{abstract}
Healthcare is seen as one of the most important areas for networked enterprise applications \& services. Much effort is put into IT to achieve better efficiency \& quality of service. Especially the fields of Patient general \& preventive Education are associated with desirable outcomes like greater satisfaction and compliance of the patient. This work addresses the issue of more efficient handling of doctor-patient relationship. HealthBot.Net uses Agent technology to extend patient-doctor relationship beyond the physical \& logistical limitation offace-to-face consultations. Work was done at a sample of 47 Patients educated before Catheter Ablation. Objective was to give patients possibility to let them (pre)access \& enter information speaking "natural" language and evaluate the impact. Second objective was to provide a conversation overview \& screening objective for the doctor.
\end{abstract}

\section{Introduction}

Starting in the past years the application scenario in the health care industry changes dramatically into a network driven enterprise with integrated processes and intercomponent data sharing. This resulted in a need for the decentralization of the computational capabilities and a deep modification of the systems architecture [1].

The therapeutic value of the patient-doctor relationship has long been recognized: The creation of an integrated healthcare workflow must include the patient.

\subsection{Background}

As for that, Patient involvement in the healthcare process is important to all attempts - specially but not only in preventive care and guidance of the chronically ill. As shown in different studies, this takes deep impact on the improvement of health care quality and patient satisfaction [2] associated with several desirable outcomes, including greater satisfaction, increased adherence to treatment, and positive treatment outcomes [3]. As a special, healthcare involves wide semantic differences due to the diversity of terminology used by different interacting disciplines. This has led to more than twenty different types of standard bodies in this area. This is the reason, why solutions in healthcare often try and have to seek for solutions preferring a so called "Agents-like" Technology, which means Software able to act in a specific environment in order to fulfill their design specifications. Agent-like Software can respond to external changes, and communicate when needed with other Agents using an agreed language [4]. This approach helps design complex systems, where different intercommunicating functionalities are implemented using dynamic and distributed components.

\subsection{Objective of work}

This paper proposes the application of agent technology to the integration of the medical treatment process illustrated through a typical doctor-patient relationship. The Work was recently developed by Elsner and Mazzi [5] [6]. Focus of this paper was the evaluation of the impact of a bot-patient conversation in a blinded trial with 47 patients at the Heart Center Leipzig. Second objective was to illustrate the basic approach of the different Answer Types in the HealthBot.Net Engine and the Development of a special Screening / Graphical Log Functionality (see Figure 1). With the Function the system is able to provide useful screening of Patients conversations and symptoms for treating doctors.

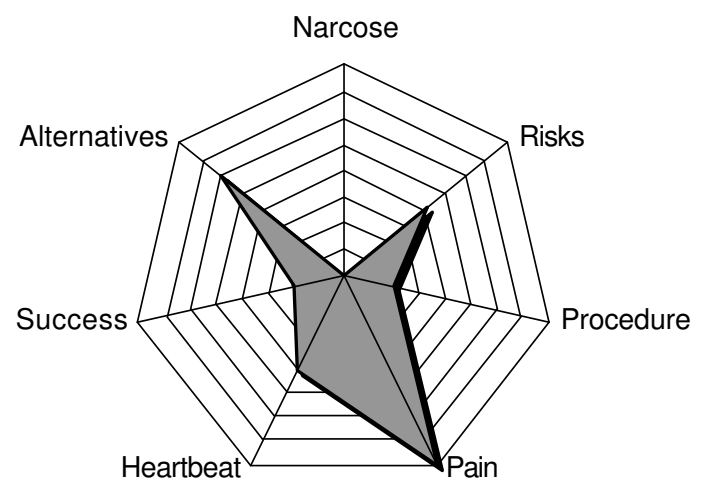

Figure 1. Radarchart of conversation topics generated from the Chat-Log after Patient conversation 


\section{Materials and methods}

\subsection{Technology used}

For the Programming of the Bot we used the Artificial Intelligence Meta Language (AIML). For the Deployment a variant of the ALICE Program D [7] and a Cocoon/Apache Server [8] were used with different modular extensions in Java and PHP.

The Software consists of a normal client-server architecture. The webbased User frontend for Patients and Administrators is therefore capable of running on every webbrowser and/or Handheld Browser.

The Core Architecture is built modular in a class oriented manner with the PHP and Java / ALICE Program-Code. Figure 2 gives a schematic overview of the different components communicating in a pipeline-manner. User Input is preprocessed over simple PHP-Scripts, which replot the Input after stripping special characters and unnecessary wordings and then restructure it for the needs of the core ALICE Engine [7]. With the special Extensions written, the ALICE Engine itself processes the code in two steps: First the input is abstracted and "synonimized" from the "Synonyms and Typos" Part of the Database. Then the engine runs the abstracted dataset against two standard checks.

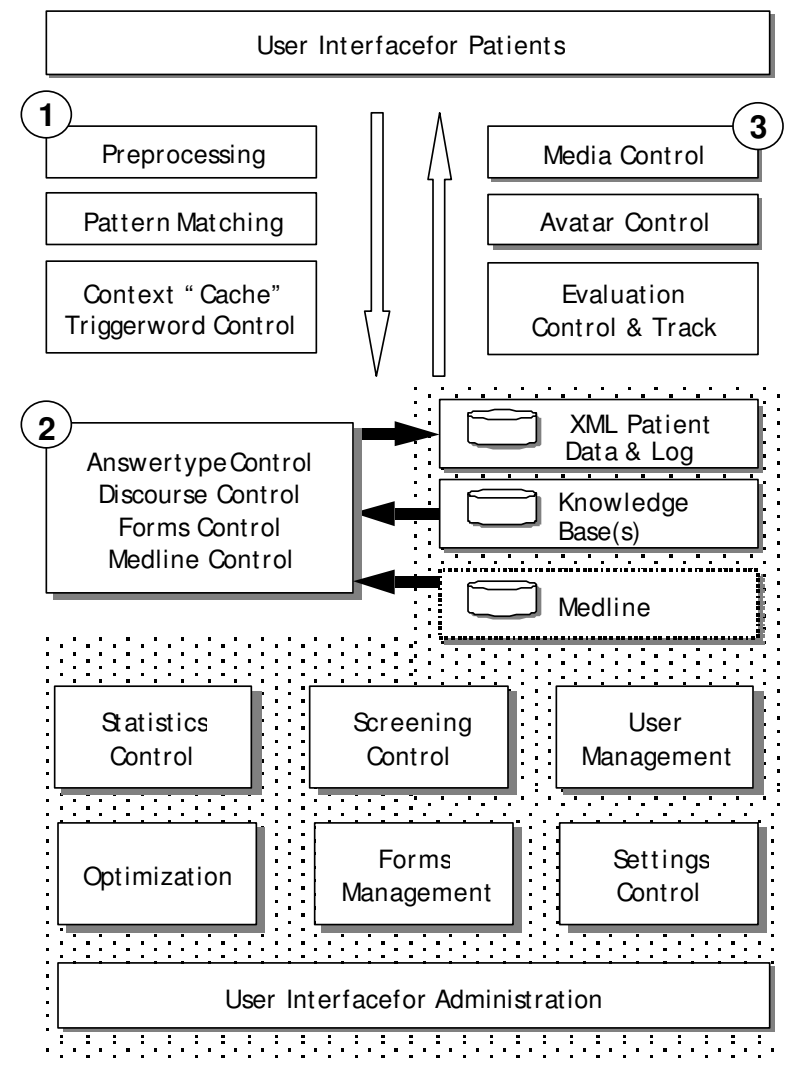

Figure 2. Schematic Overview of System Architecture with Patient- and Administration Interface

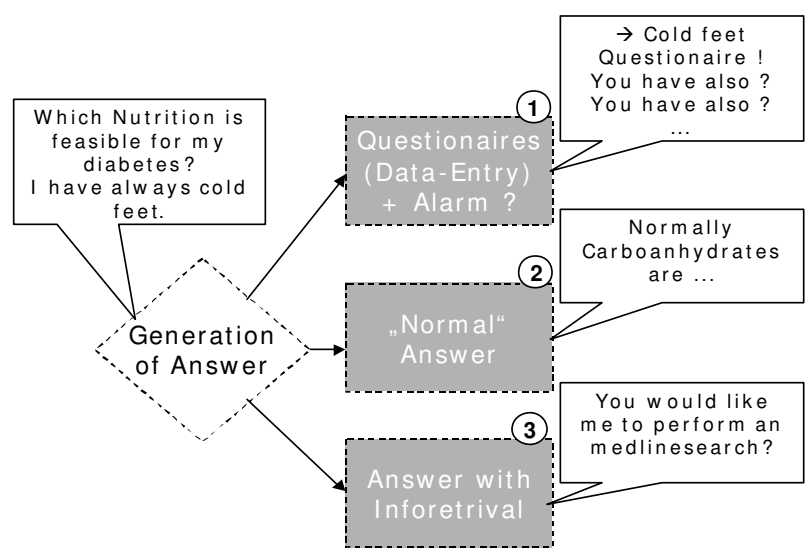

Figure 3. Overview of different Answertypes of the System with given examples

The first check is for any possibilities of context information from the preceding user inputs - e. g.:

User: „Will I have any pain?“

Bot: You mean Pain during or after the operation?

User: „After it.“

Bot: "After the Operation you will ..."

The second check is for any "trigger words" for questionnaires or external requests to the Medline - e.g.:

User: "I have always cold feet."

Bot: "Let me give You a standard questionnaire ..."

After that, adequate answers are chosen: Figure 3 gives an overview of the different Answertypes being generated. With the Answers also Actions - like raising a special "alarm flag" for the Doctor or just displaying a media element or "moving" the Avatar - are chosen and pushed to the webfronted and logged in XML-Format. The Logs serve for the chat-overviews like shown in Figure 1 done by a special Parser plotting a Radarchart of the conversations topics and/or detected symptoms. Depending on the Values already assigned to the User, an intermittend discourse and forms-management "Answer Control" Module takes care of "collecting" standardized forms \& data from the patient (e. g. like daily blood sugar or special yes/no questions). This feature is also closely linked to the so called "Discourse Control", which takes care of covering certain topics in the conversation - if not done by the patient, initiative is taken by the HealthBot. The Avatar itself was customized with a standard avatar 3D software. For speech output the Microsoft Agent Technology ${ }^{\circledR}$ was in-volved in the code output. At the End the - completely java / web-based - Administrator Interface lets a doctor view the Chat-Logs textually and graphically, lets him assign users / passwords and adjust settings of the HealthBot. Optimization of the Knowledgebase can be done directly from the textual Userlogs: Given or new answer topics can be assigned to different keypatterns, contextinformation and Forms \& Medline requests. 


\subsection{Content maintenance}

The Database on therapeutic catheter ablation in AVNode Re-entry Tachycardia was built by 3-steps of preinterviews with patients. For this, a pool of standard questions was setup and clustered into different Topics and subtopics.

For every topic and subtopic standard answer generation schemes for the HealthBot were built and filled in interviews with doctors. A simple Wordfile with Macros was used in doing this. For the consecutive maintenance of the databases and logical rules a webinterface and an additional javainterface is used.

After the HealthBot was tested with 7 patients, the content and rules were freezed for the duration of the Trial.

\subsection{Evaluation methods}

Evaluation was done in two groups with $(\mathrm{n}=24)$ and without $(n=23)$ the Bot-Interview. Patient questionnaires were given during the talk with the HealthBot over the Bot-Interface.

After each Answer of the Bot, the Patient had to grade from 1 to $6(1=$ perfect to $6=$ question not answered at all). Doctor questionnaires were given after each real interview - independent from the patient having had the Bot-Interview or not.

The doctor was not informed, if the patient had the Bot-Interview or not - that way the Trial was blinded for the doctor. The Overview chart for the doctor was not used and is subject of investigation in further studies.

\section{Results with $\mathbf{4 7}$ patients}

Our Results with a Cohort of 24 Patients showed good acceptance of the Bot-Conversation. Due to the the underlying Illness, population was relatively young with an average age of 42. Patients were randomly chosen, then told about the Trial. No patient declined participation.

In Figure 4 the results from the Grading of the BotAnswers is shown: $43,5 \%$ of the Bot-Answers were graded useful / good, 35,8 \% of the answers were graded bad or not matching.

Patients having the Bot-Coversation asked 4.6 questions in average, patients without Bot-Interview asked 3.2 questions in average. There were no significant changes in the "Detail of Question" by the Patient, and no significant changes in being informed about the Procedure.

The only significant change in our group was in the number of questions being asked by the patient: The BotInterview Patients asked 1,4 Questions more.

Figure 5 shows the Results of the detailed questionnaire to the doctor.

Overall the whole Patient-Population seemed to be informed above average.

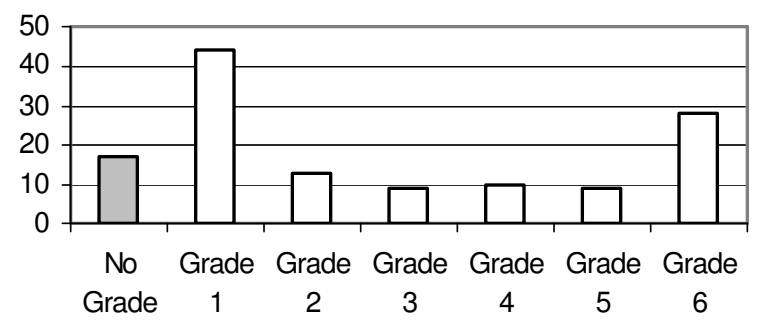

Figure 4. Graded Bot Answers by Bot-Group ( $\mathrm{n}=24)$ : $29 \%$ were not answered correct, $13 \%$ were not graded.

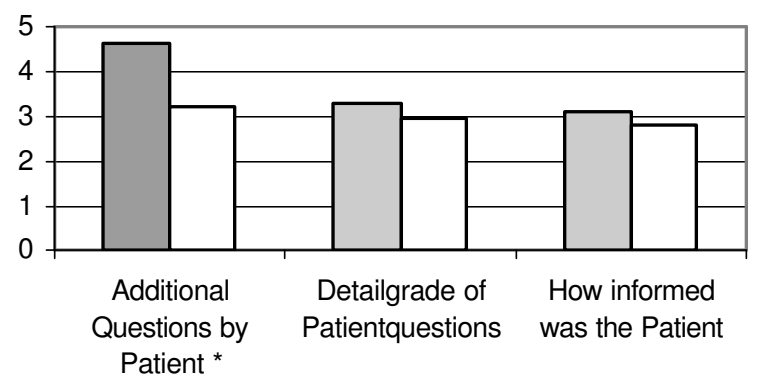

Figure 5. Result of Questionaires to Doctors. (Shaded cols = Pat. with Bot-Interview, white cols $=$ Pat. without Bot-Interview). ${ }^{*}=$ Significant change marked dark.

\section{Discussion}

\subsection{General project approach}

Still Patients value most the information provided to them by their doctor [9] and in today's society individuals will have to take greater responsibility for their healthcare. So the general approach of the project of fitting medical information into the ongoing dialogue between doctor and patient seems very feasible. The Project may be interpreted to serve the "modern" and future patient, which will actively demand more information from their doctors [10]. A further enhancement may be the used Avatar: Psy-chological studies have determined that individuals attribute anthropomorphic characteristics to the machines they interact with. This behavior can be leveraged when dispatching a HealthBot to a patient. Furthermore the developed graphical overview Radarchart could be of help in tracking changes in symptoms / conversation topics. Investigations showed this form of information being most efficient and good accepted [11].

\subsection{Interpretation of results}

With this work a so called HealthBot was developed. The implementation makes it flexible for further topics and accessible via web-based technology in a server / client architecture. This Architecture is ideal for diverse 
distribution channels in different diseasemengement settings. An Evaluation in a blinded Trial with 47 patients showed, that for the limited topic of "therapeutic catheter ablation in AV-Node Re-entry Tachycardia" a good result in the quality of Bot-Answers can be achieved with the tools. A further investigation on the doctor-patient interviews following a Bot-Interview showed a significant change only in the number of questions being asked by the patient - no significant rise of the Patients in being "more informed" could be tracked. This may result from the chosen population, which seemed overall well informed above average. A more detailed questionnaire to the doctor or a bigger cohort maybe necessary to see significant changes in this property. Also longitudinal Trials on a population of chronically ill seems to be a further feasible investigation, as with the current work only short-term effects were tested.

What could be obtained with this Trial was a clear result in the number of questions asked by Patients having had the Bot-Interview: Significantly more Questions were asked by that population. Together with the result patients not being informed any worse, one may interpret that Patients get more interested in the topic itself and their Healthcare. This would show a clear effect of a tighter inclusion of the patient in the healing process.

\subsection{Conclusion}

The technology showed to integrate the patient in the Process of HealthCare can have high impact on this Process: A review of 22 studies by Stewart et Al [13] indicated that positive effect of communication on actual patient health outcome such as pain, recovery from symptom, anxiety, functional status, and physiologic measures of blood pressure and blood glucose.

Future developments in healthcare technology should focus on improving this positive trend of patient integration. To this purpose, the HealthBot Technology can play a key role in facilitating patient-doctor communication. Furthermore from a perspective of HealthCare ser-vices, presence of a HealthBot-Interaction is an oppor-tunity for proactive healthcare and taking care of the chronically ill. Patients will often experience symptoms long before they visit a doctor. In fact, studies indicate that patients with a history or a gradual progression of symptoms will present to the doctor later than patient with acute attacks and generally doctors perceive a greater need for investigation than patients [12]. Furthermore, patients will often seek information about symptoms to decide on whether to see a doctor.

The described technology with "Discourse Management" and the Management of Forms and Screening Mechanisms combined with Radarcharts for quick overview seems to be ideal for this task. Arguably, patients with potentially serious medical conditions could be detected earlier, usually improving prognosis.
Overall our results encourage our approach in training a HealthBot for specific patient education. Our first version seems to stimulate Patients for a deeper and more detailed understanding. For future aspects Patient monitoring and continuous health education could be feasible with the provided tools and should be topic of further investigations.

\section{References}

[1] Lanzola $G$ et al. A framework for building cooperative software Agents in medical applications. In: Artificial Intelligence in Medicine 1999;16:223-249.

[2] Kim J et al. An empirical study of the Health Status Questionnaire System for use in patientcomputer interaction. In: Proceedings / AMIA Annual Fall Symposium 1997;86-90.

[3] Tennstedt SL. Empowering older patients to communicate more effectively in the medical encounter. In: Clinics in Geriatric Medicine 2000; 16(1):61-70

[4] Della Mea V et al. Internet Agents for telemedicine services. In: Medical Informatics 1999; Vol. 24/3:181188.

[5] C Mazzi, P Ganguly \& M Kidd. Healthcare Applications based on Software Agents. In: Health and Medical Informatics 2001; 10: 136-140.

[6] Elsner CH, Meyer P, Helbing K, Hindricks G: Natural speaking Software Agents for patient education before rhythmologic intervention: Preliminary results from an AIML prototype. HealthCare meets Medical Informatics \& Innovations, HealthCare MMII Congress from 20 - 22th October 2002 in Edinburgh

[7] See URL http://www.alicebot.org

[8] See URL http://www.apache.org

[9] A.Wolf, H.Kottkamp, S.Menz and Ch.Elsner. Aspects of designing Cross-platform Information Portals for eHealth Applications: Results from a 235 patient survey end of year 2000. World Congress in Internet an Medicine November 2001 in Udine

[10] Griffin JP and Griffin JR. Informing the patient. In: Journal of the Royal College of Physicians of London 1996; 30(2): $107-11$

[11] van Bemmel JH. Criteria for the acceptance of decisionsupport systems by clinicians. In: lessons from ECG interpretation system; Proceedings of the AIME'93 Conference 1993: 7-10

[12] Peay MY and Peay ER. The evaluation of medical symptoms by patients and doctors; J Behav Med 1998 Feb; 21(1):57-81

[13] Stewart M. Evidence on patient-doctor. In: communication; Cancer Prev Control 1999 Feb;3(10):25-30

Address for correspondence.

Christian Elsner

Thomasiusstr. 9

04109 Leipzig

Germany

Ch.Elsner@gmx.de 